\title{
Curcumin/Chondroitin Sulfate/Hyaluronic Acid/Quercetin Soft Gels
}

National Cancer Institute

\section{Source}

National Cancer Institute. Curcumin/Chondroitin Sulfate/Hyaluronic Acid/Quercetin Soft

Gels. NCl Thesaurus. Code C156174.

An orally available preparation containing curcumin, quercetin, hyaluronic acid, and chondroitin sulfate with potential radioprotective activity. Upon administration, the glycosaminoglycans may improve barrier function and reduce inflammation and irritation in the genitourinary tract caused by radiotherapy. 